\title{
La colección de tipos de plantas vasculares del Herbario Nacional de Chile (SGO). Análisis histórico, temporal y espacial
}

\section{The vascular plants type collection of the Chilean National Herbarium (SGO). Historic, temporal and spatial analysis}

\author{
Mélica Muñoz-Schick ${ }^{1}$, Vanezza Morales ${ }^{2}$ \& Andrés Moreira-Muñoz ${ }^{2}$ \\ ${ }^{1}$ Museo Nacional de Historia Natural, Casilla 787, Quinta Normal, Santiago, Chile. \\ 2Instituto de Geografía, Pontificia Universidad Católica de Chile, Vicuña Mackenna 4860, Macul, Santiago, Chile. \\ mmunoz@mnhn.cl
}

\begin{abstract}
RESUMEN
Se realiza un análisis de la colección de especímenes tipo de plantas vasculares del Herbario Nacional de Chile (SGO), aportando antecedentes a su historia, a la distribución temporal y espacial de los ejemplares, los colectores y descriptores más importantes junto con la validez de los nombres de los taxones analizados al día de hoy. Se analizaron 6.555 ejemplares, los que fueron recolectados en un período de 182 años (1828-2010). La mayoría de éstos se concentran espacialmente en las regiones de Atacama, Coquimbo y Metropolitana. En cuanto a su validez taxonómica actual, se analizaron 4.236 nombres de taxones, de los cuales el 61\% mantienen a lo menos el género y el 20,4\% el nombre completo de la especie o categoría infraespecífica con que fue descrito originalmente. Como descriptor más importante se destaca R.A. Philippi, con 3.359 nombres de taxones dentro de la colección, mientras que junto con F. Philippi destacan como colectores de un gran número de los ejemplares tipo.
\end{abstract}

Palabras clave: Colección tipo, plantas vasculares, Herbario SGO, Chile.

\begin{abstract}
The vascular plants type collection of the SGO Herbarium, Chile, was studied, including information about its history, temporal and spatial distribution of the specimens, prolific collectors and descriptors, together with the revision of the valid names up to date. The analyzed specimens reach a number of 6,555, collected in a lapse of 182 years (1828-2010). A great proportion of the specimens was collected in the Atacama, Coquimbo and Metropolitan regions. According to the names validity, from 4,236 specimens, $61 \%$ retains at least the genus name and $20.4 \%$ retains the complete name of its original description. R.A. Philippi appears as the most prolific plant descriptor, with 3,359 taxa, while together with F. Philippi they act also as collectors of a high number of type specimens.
\end{abstract}

KeYwords: Type collection, vascular plants, SGO Herbarium, Chile.

\section{INTRODUCCIÓN}

Las primeras colecciones de plantas que componen el actual Herbario Nacional (SGO) datan de 1828, y corresponden a cerca de 400 ejemplares del italiano Carlo José Bertero, quien recolectó en Chile entre 1828-1830 (Muñoz-Schick 1999). Al crear Claudio Gay el Gabinete de Historia Natural en 1830, con colecciones propias y las de Bertero, sentaría las bases del Herbario SGO. El Herbario se aloja en el Museo Nacional de Historia Natural, que ocupa el histórico edificio al interior de la Quinta Normal desde 1876, gracias a la gestión de su entonces director Rodulfo Amando Philippi
(1808-1904). El eminente naturalista alemán creó en 1889 la Sección Botánica, la cual quedaría inmediatamente a cargo de su hijo Federico Philippi (1838-1910). En esa época la colección ya comprendía cerca de 32.150 ejemplares: a) los 400 ejemplares de Bertero; b) cerca de 1.700 duplicados de Claudio Gay (Muñoz-Schick 2007); c) más de 50 ejemplares de Vicente Bustillos, padre de la farmacia chilena, quien recolectó entre 1843-1863; y d) los ca. de 30.000 ejemplares de R.A. Philippi y sus colaboradores, recolectados a lo largo de Chile y países limítrofes entre 1851 y 1889 (MuñozPizarro 1960).

Una parte importante de las colecciones históricas 
mencionadas constituyen ejemplares tipo (i.e. ejemplares sobre los que se basa la descripción de un taxón), los cuales han sido sistematizados a partir de la década del '40. En esa época, el Museo Nacional contaba con una gran cantidad de material que no se encontraba montado según las normas internacionales, por lo que las labores de reconocimiento y organización dentro del herbario se hacían difíciles. Además se corría el riesgo de pérdida o destrucción del material. Por ello se diseñó un plan de organización del herbario en el marco de un programa de cooperación entre el Departamento de Genética y Fitotecnia del Ministerio de Agricultura y del Museo Nacional de Historia Natural. La ordenación del herbario estuvo a cargo del ingeniero agrónomo Carlos Muñoz Pizarro (1913-1976), lográndose entre 1942 y 1946 la revisión de un total de 68.244 ejemplares (Muñoz-Pizarro \& Pisano 1950), a los cuales se les incorporó la etiqueta correspondiente y se les comparó con las descripciones originales. De este modo fueron separados los ejemplares tipo de las colecciones generales de plantas nativas y extranjeras.

En 1960, Muñoz Pizarro estimaba la cantidad de tipos en 3.111 ejemplares, lo que posicionaba a la colección como una de las más importantes en América Latina, ya que poseía el $83 \%$ de los tipos de plantas chilenas descritas por R.A. Philippi (Muñoz Pizarro 1960).

Como toda colección biológica, la colección de tipos se ha ido incrementando con el tiempo, lo cual plantea el desafío de su continua revisión, puesta al día y actualización. En el año 2009 se dio curso a la digitalización de los tipos del Herbario SGO, lo que contempló la creación de la Base de Datos de la Colección. Dicha base reúne información de la etiqueta y del ejemplar, con la que ha sido posible precisar, entre otros, colector, lugar de recolección, y evaluar si el ejemplar corresponde o no a un tipo. Gracias a ello es pertinente y factible exponer en la presente publicación: a) los datos de la colección completa; b) análisis temporal de los datos (época en que se recolectó el mayor número de ejemplares tipo); c) análisis espacial (número de ejemplares tipo recolectados por área); d) reconocimiento de los colectores y descriptores más importantes y e) cálculo de la validez de los nombres de los tipos nomenclaturales.

\section{MATERIALES Y MÉTODO}

\section{REVISIÓN DE LA BASE DE DATOS}

La base de tipos analizada consta de 6.555 registros, y considera sólo aquellas especies nativas de Chile o de países limítrofes (incluyendo Brasil). Se han obviado 203 ejemplares pertenecientes a la colección de Sellow, de especies exóticas de la familia de las Mirtáceas. De la base de tipos se revisaron los campos: a) colector; b) fecha de recolección de los ejemplares; c) nombre del taxón (especie, subespecie, variedad o forma) asociado a cada tipo; d) descriptores de estos taxones y sus respectivos nombres válidos actuales. La información fue contrastada con las publicaciones de Muñoz Pizarro (1960), Muñoz-Schick (1971,1973, 1999, 2007), Muñoz-Schick \& Prina (1987), Marticorena \& Rodríguez (1995) y Zuloaga et al. (2008). En el caso de algunos tipos que no aparecen mencionados en las publicaciones anteriormente citadas fue necesario recurrir a estudios taxonómicos actuales para determinar la validez de los nombres (e.g. Klingenberg 2007 para Haplopappus Cass.).

\section{DISTRIBUCIÓN TEMPORAL}

Mediante la confección de una curva acumulada de valores se pudo determinar cuál fue el período de tiempo y año con más ejemplares tipo recolectados. Se utilizaron 5.342 registros de la base de datos que contaban con año de recolección $(81,5 \%$ del total).

\section{DisTRIBUCIÓN ESPACIAL}

Se analizó la distribución de los tipos para el segundo nivel político-administrativo de cada país (regiones para Chile, provincias para Argentina y departamentos para Bolivia y Perú). Las islas oceánicas de Chile pertenecen administrativamente a la región de Valparaíso, pero han sido tratadas aparte, ya que corresponden a territorios espacialmente discontinuos.

Si bien cada ejemplar está asociado a un país y región en la base de datos, no se contaba con las coordenadas de la localidad específica, por lo que hubo que georreferenciar las localidades. Se utilizó las siguientes fuentes de información: Instituto Geográfico Militar (IGM, 1984), Riso Patrón(1924), San Román (1892) y la Base de Datos GeoNames (www. geonames.org). Para asignar las coordenadas a localidades históricas de ciertos recolectores fue necesario analizar el itinerario de sus viajes (e.g. Hirth 1886, Ibar 1878, F. Philippi 1865, 1885, 1886, R.A. Philippi 1862, 1889).

Los registros que no fueron georreferenciados obedecen a cuatro razones: a) no fue posible encontrar las localidades (e.g. Cuesta de La Arena en Cordillera de Talca); b) amplitud de la zona especificada (e.g. Cordillera de Chillán, Araucanía, Pampa de Patagonia); c) repetición de nombres de localidades para una misma región (e.g. dos Hacienda Mercedes en Región Metropolitana); d) repetición de nombres de localidades para distintas regiones (e.g. Valle Hermoso en Región del Maule y Biobío). En los dos últimos casos, los registros no cuentan con los antecedentes necesarios para determinar cuál es la localidad correcta.

\section{COLECTORES MÁS IMPORTANTES}

Se utilizó la información de 6.460 ejemplares que poseen colector conocido en la base de datos $(98,6 \%$ del total). Se consideraron los diez colectores con mayor número de ejemplares tipo, que además reúnen un número mayor que 100. 
Los ejemplares de R.A. Philippi y F. Philippi se consideraron de forma conjunta, ya que sus letras manuscritas son muy semejantes (Taylor \& Muñoz-Schick 1994), y en una buena parte de sus etiquetas no se explicita quién de los dos fue el recolector responsable. Sólo en algunos de estos casos fue posible determinar a quién correspondían exactamente. Para esto se revisó: a) el estudio crítico de los tipos nomenclaturales de R.A. Philippi y F. Philippi (e.g. Oxalis brevis Phil. fue recolectado por F. Philippi) (Muñoz-Pizarro 1960: 78); b) las publicaciones de sus viajes (e.g. Haplopappus pectinatus Phil., fue recolectado en Chonchoral por R.A.Philippi) (R.A.Philippi 1889: 283)

DESCRIPTORES MÁS PROLífICOS

Para obtener el número de taxones por cada descriptor se contaron los nombres de los tipos, sin considerar el número de ejemplares con un mismo nombre científico (e.g. el nombre Haplopappus australis Phil. sólo se contabilizó una vez, aunque tiene varios ejemplares entre lectotipo (1), isotipo (1) y sintipos (2)). Por último, se contaron los nombres de los tipos con un mismo descriptor, destacando en los resultados los 20 primeros.

VALIDEZ ACtUAL PARA LOS NOMBRES DE LOS TIPOS A cada nombre de tipo único (ver punto anterior) se le asignó una de tres categorías posibles: 1) Aceptado; 2) Sinónimo; 3) No resuelto (por falta de estudios que lo señalen, se desconoce si el nombre del tipo es válido o sinónimo de algún otro taxón) (e.g. Asarca inaequalis Phil.).

Finalmente, se calculó el nivel de representatividad de la colección de tipos con respecto al número total de especies de la flora vascular chilena según Moreira-Muñoz (2011). Para el cálculo se consideró las categorías 1) Aceptado y 2) Sinónimo; obviamente no se incluyen los tipos de nombre no resuelto (ver Apéndice).

\section{RESULTADOS}

DistribuCIÓN TEMPORAL

Hasta julio de 2011, la colección de tipos del herbario SGO consta de 6.555 ejemplares, los que fueron recolectados en un período de 182 años (1828-2010).

La acumulación temporal de los ejemplares (Fig. 1) muestra que 4.809 tipos ( $90 \%$ del total considerado) fueron recolectados hasta 1890 (primeros 62 años).

El período en que se aprecia la mayor cantidad de ejemplares es 1850-1890. Este segmento de tiempo concuerda con las labores de R.A. Philippi y F. Philippi a cargo del Museo Nacional, quienes dieron un gran incentivo a las campañas de terreno (Muñoz-Schick 1991).

Por otro lado, el año con mayor cantidad de ejemplares es 1885 (706); la mayor parte de este material se puede asociar a dos viajes, ambos encabezados por F. Philippi. El primero fue la expedición a la región de Tarapacá (Norte de Chile) entre los meses de enero y abril. Este viaje abarcó el territorio desde Copiapó a Tarapacá pasando por la Puna de Atacama (los ejemplares de este viaje fueron recolectados por F. Philippi y Carlos Rahmer). El segundo viaje fue principalmente a la costa de la región de Atacama durante los meses de septiembre y octubre (los colectores más importantes de este viaje fueron F. Philippi, Sr. King y Rosario Godoy de Collao). Con respecto al nombre de pila del Sr. King no existe claridad; en la mayoría de las descripciones de R.A. Philippi se menciona a Tomás King (e.g. Alstroemeria kingii Phil. Anal. Univ. Chile 43: 548. 1873), mientras que en la publicación del viaje se menciona a los señores Juan King y Thomas Fling (F. Philippi 1886: 8); nuestra opinión es que F. Philippi confundió el nombre de pila de estas personas en la publicación y que el nombre correcto sería Thomas.

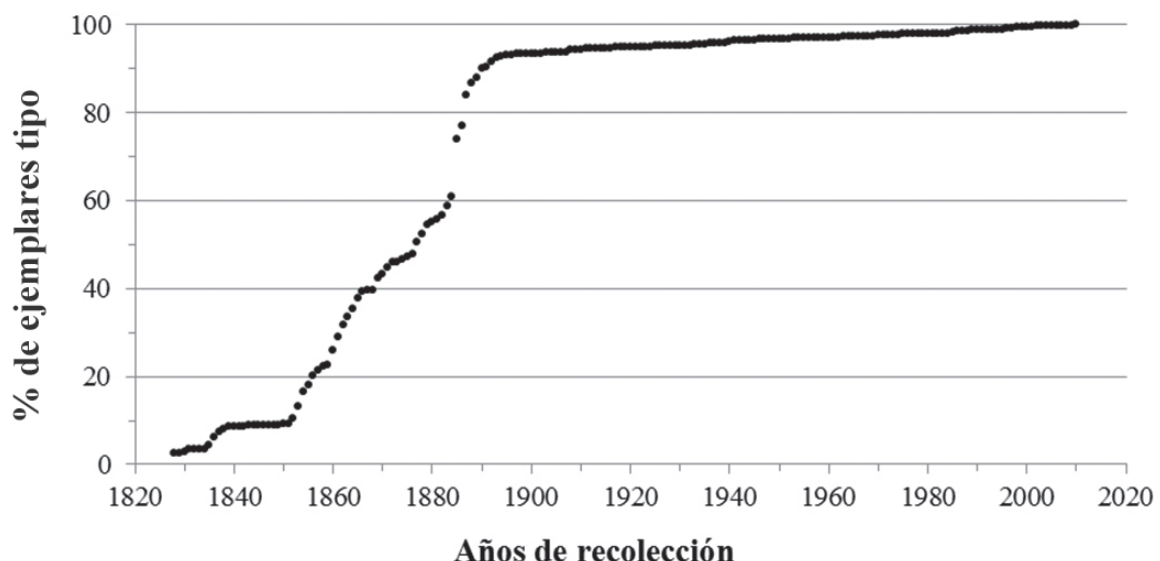

Figura 1. Curva acumulada de ejemplares tipo en Herbario SGO.

Figure 1. Accumulation curve of type specimens in SGO Herbarium. 
DISTRIBUCIÓN ESPACIAL

Se logró agregar coordenadas geográficas a 4.614 ejemplares (70,4\% del total). El 92\% de los ejemplares fue recolectado dentro de los límites actuales de Chile; el porcentaje restante corresponde a ejemplares herborizados en localidades de Argentina (479), Bolivia (31), Perú (12) y Brasil (1) (Tabla I).

En Chile, las regiones con mayor número de ejemplares tipo son tres: Coquimbo (765 ejemplares), Atacama (660) y Metropolitana (657) (Tabla I, Fig. 2a). El caso de la región Metropolitana es esperable pues ello se asocia a la temprana ocupación histórica del territorio, lo que queda en evidencia con las primeras colecciones de Bertero. La concentración de los tipos en las regiones de Coquimbo y Atacama se explica tanto por las recolecciones de Claudio Gay como por las de los Philippi (padre e hijo) y sus colaboradores (e.g. Francisco San Román, Federico y Guillermo Geisse, Luis Landbeck y Herman Volckmann) (Tabla II). Por el contrario, la baja presencia de tipos en el extremo norte del país (actual región de Arica y Parinacota), se explica debido a que en esa época (previo a 1879) no se habían anexado aún dichos territorios a Chile.

La paulatina disminución de la cantidad de tipos hacia las regiones australes, específicamente la Región de Aysén, se podría explicar por las difíciles condiciones de accesibilidad de hace dos siglos y que existen aún hoy en día.

En el caso de Argentina la mayoría de los ejemplares corresponden a las provincias de Catamarca, Mendoza y Neuquén. En la provincia de Catamarca, la mayoría de los ejemplares fueron recolectados por F. Philippi en 1885 durante el viaje a Tarapacá (Muñoz-Schick \& Prina 1987), zona que en ese entonces y hasta 1899 fue reclamada por Chile (Espinosa 1958). En la provincia de Salta la recolección de F. Philippi de 1885 no fue tan rica como la de Catamarca, ya que en ese tramo de la expedición "la vegetación era muy escasa en todo el camino" (F. Philippi 1885: 141). La representación de los sitios de recolección muestra claramente las rutas seguidas por los recolectores (Fig. 2b).

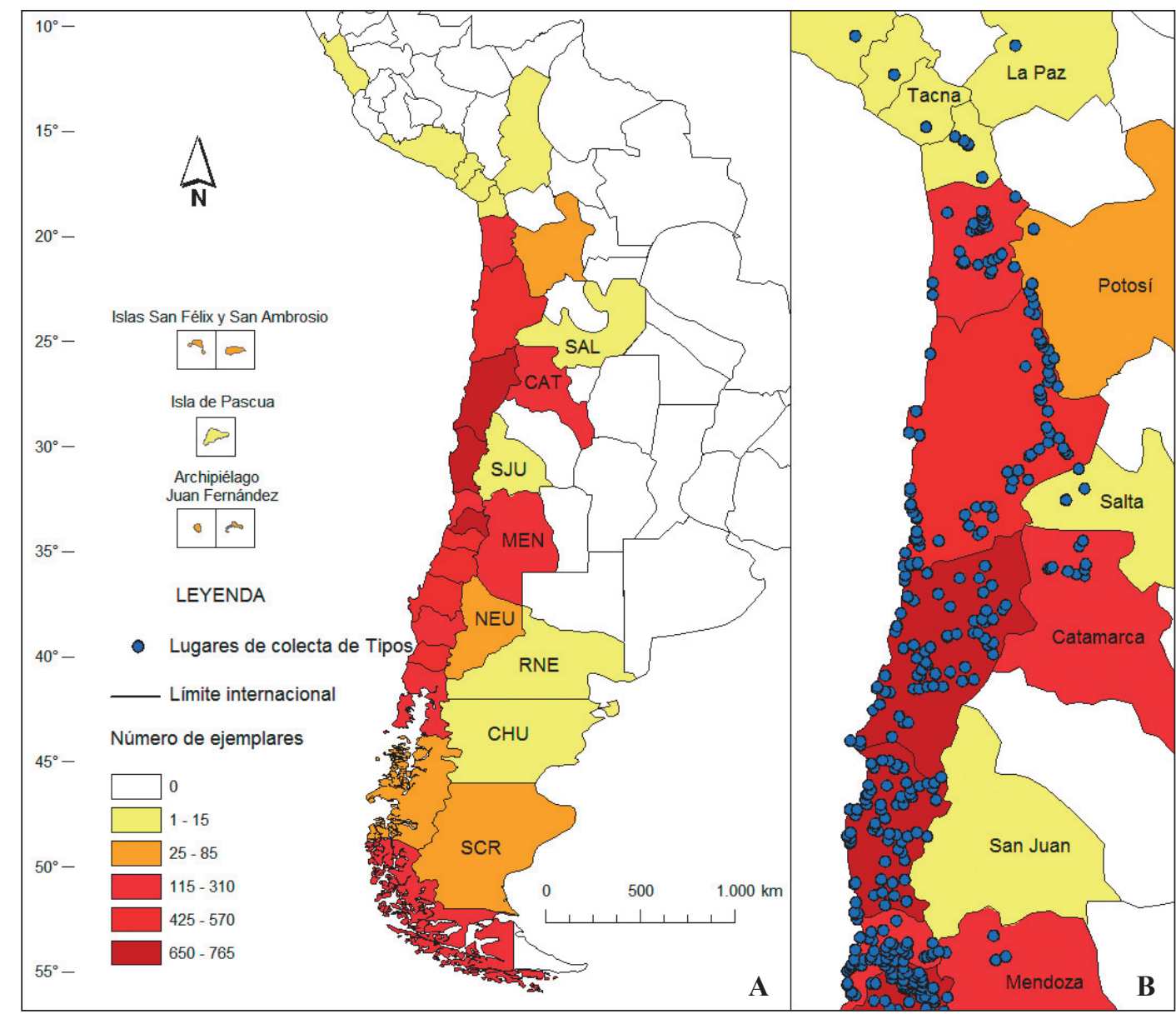

Figura 2. A. Número de ejemplares tipo de Chile y países vecinos, por segundo nivel político administrativo. B. Distribución de los ejemplares tipo en la zona norte de Chile, Argentina y sur de Bolivia.

Figure 2. A. Number of type specimens of Chilean and neighboring countries at the second political level. B. Distribution of type specimens of northern Chile, Argentina and southern Bolivia. 
TABLA I. Número de ejemplares tipo por país, región, departamento o provincia en Herbario SGO (Países ordenados alfabéticamente; regiones, provincias y departamentos ordenados de norte a sur).

TABLE I. Number of type specimens for country, region, department or province in SGO Herbarium (The countries are presented by alphabetic order; regions, provinces and departments are ordered from north to south).

\begin{tabular}{|c|c|}
\hline PAís, REGIÓN, DEPARTAMENTO O PROVINCIA & NÚMERO DE EJEMPLARES TIPO \\
\hline Argentina & 479 \\
\hline Salta & 1 \\
\hline Catamarca & 118 \\
\hline San Juan & 1 \\
\hline Mendoza & 216 \\
\hline Neuquén & 78 \\
\hline Río Negro & 9 \\
\hline Chubut & 8 \\
\hline Santa Cruz & 26 \\
\hline Islas Malvinas & 4 \\
\hline No determinado & 18 \\
\hline Bolivia & 31 \\
\hline $\mathrm{LaPaz}$ & 1 \\
\hline Potosí & 29 \\
\hline No determinado & 1 \\
\hline Brasil & 1 \\
\hline Chile & 6.032 \\
\hline Arica y Parinacota & 9 \\
\hline Tarapacá & 179 \\
\hline Antofagasta & 549 \\
\hline Atacama & 660 \\
\hline Coquimbo & 765 \\
\hline Valparaíso & 435 \\
\hline Metropolitana & 657 \\
\hline Libertador General Bernardo O’Higgins & 453 \\
\hline Maule & 426 \\
\hline Biobío & 567 \\
\hline Araucanía & 191 \\
\hline Los Ríos & 443 \\
\hline Los Lagos & 207 \\
\hline Aysén del General Carlos Ibáñez del Campo & 42 \\
\hline Magallanes y la Antártica Chilena & 156 \\
\hline No determinado & 293 \\
\hline Perú & 12 \\
\hline Lima & 2 \\
\hline Arequipa & 2 \\
\hline Moquehua & 1 \\
\hline Tacna & 3 \\
\hline No determinado & 4 \\
\hline Total & 6.555 \\
\hline
\end{tabular}


El resto de las provincias argentinas fueron exploradas varias veces por distintos naturalistas, algunos con fines botánicos o de reconocimiento geográfico. Por ejemplo, los tipos recolectados en la provincia de Mendoza fueron traídos por Federico Albert, Augusto Borchers (Baños del Inca $=$ Puente del Inca), Wenceslao Díaz (Portillo de los Piuquenes, lado de Mendoza), Luis Goldsack, Juan Isern, Max Landbeck (entre Santiago y Mendoza $=$ Portillo de los Piuquenes), Federico Leybold, Pablo Ortega y Edwin Reed, todas éstas entre los años 1854 y 1890.

Con la revisión de las publicaciones de los viajes de algunos de los recolectores de ejemplares tipo, se pudo localizar exitosamente varios lugares, entre los que se cuentan aquellos que han cambiado de nombre (e.g. Chonchoral = Quinchamalí) (R.A. Philippi 1862) y de los que se desconocía su posición dentro del Cono Sur (e.g. Isma, Napa, Cana, Caishape, lugares visitados por F. Philippi durante 1885 en su viaje a la provincia de Tarapacá y que se localizan cerca del límite con Bolivia en la región de Tarapacá y el norte de Antofagasta) (F. Philippi 1885). De otro modo y utilizando las localidades y fechas presentes en las etiquetas de los colectores se reconstruyeron algunos viajes sin publicaciones; esto permitió ubicar correctamente lugares donde la variación de la escritura lo impedía (e.g. Huahuim $=$ Hua-Hum y Pucaullu $=$ Pocaollo, hasta ahora ambas localidades habían sido erróneamente citadas para nuestro país. Se reconoce este error luego de haber estudiado el viaje de Otto Philippi en el verano de 1887, donde recorrió la cordillera de la región de Los Lagos pasando al lado argentino en la provincia de Neuquén). Gracias a este último resultado se ha podido explicar que algunas especies no se encuentren efectivamente en Chile, y que en el pasado fueron citadas para nuestro país unicamente a través de sus tipos (e.g. Homoianthus calophyllus Phil. = Perezia calophylla (Phil.) Reiche, sólo presente en las provincias argentinas de Neuquén y Río Negro).

\section{RECOLECTORES MÁS IMPORTANTES}

En la Tabla II se muestran los diez recolectores más prolíficos (más de 100 ejemplares tipo cada uno), junto con las fechas y las áreas en que recolectaron el material. Se ha incluido la cita de trabajos particulares, de modo que el lector pueda profundizar en los itinerarios de los recolectores.

Todas las personas mencionadas en la Tabla II, a excepción de C. Gay y C. Bertero, fueron colaboradores cercanos de R.A. Philippi. Por esta razón algunos de sus tipos fueron dedicados a ellos y llamados según sus apellidos. El último en la lista de colectores es Otto Philippi (hijo mayor de F. Philippi) a quien se considera colaborador dado que todos sus ejemplares (tipos y de la colección general) fueron identificados por su padre o por el abuelo.

Nos parece pertinente aclarar aquí la existencia de dos recolectores con apellido Geisse dentro del herbario
SGO, tanto en la colección general como en la de tipos. Los ejemplares más antiguos corresponden a Federico Geisse hijo ("Fed. Geisse fil." en las etiquetas). Todos sus ejemplares fueron recolectados en la región de Atacama (Quebrada de Puquios) en 1865, mientras que Guillermo Geisse (Guillermo o William en las etiquetas) recolectó en las regiones de Atacama, Coquimbo y Valparaíso entre los años 1880 a 1896.

El resto de los recolectores tienen menos de 100 ejemplares tipo, siendo el primero en la lista Wilibald Lechler con 95 ejemplares, recolectados en Magallanes y Valdivia entre 1850 y 1854.

DESCRIPTORES MÁS PROLÍFICOS

La colección de tipos del herbario SGO está compuesta por 4.236 nombres originales; casi el $80 \%$ de ellos fueron descritos por R.A. Philippi (Phil.), ocupando el primer lugar en importancia. El segundo lugar lo ocupa C. Reiche, como descriptor individual (Reiche), mientras que el tercer lugar le corresponde al mismo Reiche, pero esta vez bajo la fórmula de descripciones asignadas a otro autor (Phil. ex Reiche).

Los 20 descriptores con más nombres de taxones en la base de datos concentran el 92\% de la colección (Tabla III), mientras que el $8 \%$ restante fue publicado por 268 autores diferentes. Varios de los 20 autores seleccionados (e.g. Steudel, Clos, Remy, Desvaux) basaron sus descripciones de plantas chilenas en ejemplares de Bertero, Gay y Lechler, los que habían sido llevados a Europa y que permanecen allí. Dentro de la colección de tipos de SGO es destacable la presencia de isotipos o duplicados de los ejemplares en que estos autores basaron sus descripciones. Aunque este hecho estaba indicado en publicaciones diversas (MuñozPizarro \& Pisano 1950; Muñoz-Schick 1991) hasta ahora no se habían contabilizado.

VALIDEZ ACTUAL PARA LOS NOMBRES DE LOS TIPOS

De los 4.236 nombres de tipos de la colección, el 61\% de ellos mantiene al menos el género al que fue asociado en la descripción original y el $20,4 \%$ el nombre completo de la categoría específica o infraespecífica con que fue descrito (Apéndice).

En la Figura 3 se presentan las diez familias con mayor número de taxones cuyos especímenes tipo están conservados en SGO, siendo la más numerosa la familia Asteraceae con un 23,9\%, mientras que Poaceae y Fabaceae ocupan el segundo y tercer lugar en importancia con 8,7 y $7,1 \%$ respectivamente.

En la actualidad, los 4.236 nombres de tipos se reducen a 2.421 especies válidas; de ellas, 2.326 corresponden a especies chilenas. La comparación de esta última cifra con las 4.295 especies que conforman la flora de Chile según Moreira-Muñoz (2011), arroja un 54\% de representación botánica de la colección de tipos. 
Tipos Museo SGO: MuÑoz-Schick, M. ET AL.

TABLA II. Recolectores con mayor número de ejemplares tipo en Herbario SGO.

TABLE II. Collectors with the highest number of type specimens in SGO Herbarium.

\begin{tabular}{|c|c|c|c|}
\hline RECOLECTOR & $\begin{array}{l}\text { NÚMERO DE } \\
\text { EJEMPLARES TIPO }\end{array}$ & AÑos DE COLECTA & ZONAS VISITADAS \\
\hline R.A. y F. Philippi & 2.535 & 1851 a 1900 & $\begin{array}{l}\text { Todas las regiones de Chile, excepto Arica- Parinacota y Aysén } \\
\text { (Muñoz-Schick \& Prina 1987) }\end{array}$ \\
\hline C. Gay & 351 & 1830 a 1841 & De Copiapó a Chiloé (Muñoz-Pizarro 1944) \\
\hline Ph. Germain & 322 & 1851 a 1895 & $\begin{array}{l}\text { Desde Valparaíso a la Araucanía (Malleco), Puerto Montt y Juan } \\
\text { Fernández; Argentina (Neuquén: Laguna Varvarco) }\end{array}$ \\
\hline L. Landbeck & 242 & 1860 a 1867 & $\begin{array}{l}\text { En las provincias de Choapa, Petorca (Los Molles), San Felipe, San } \\
\text { Antonio (Algarrobo), Santiago (Las Arañas), Colchagua y Curicó } \\
\text { (Llico) }\end{array}$ \\
\hline G. Geisse & 228 & 1880 a 1896 & En las provincias de Copiapó, Huasco, Limarí, Choapa y Petorca \\
\hline C. Rahmer & 222 & 1880 a 1887 & $\begin{array}{l}\text { Interior de la región de Tarapacá y las provincias de Biobío } \\
\text { (Antuco) y Malleco (Laguna Galletué); Bolivia (Potosí: } \\
\text { Calcalhuay) }\end{array}$ \\
\hline P. Ortega & 217 & 1865 a 1886 & $\begin{array}{l}\text { En la regiones de Atacama, Coquimbo, Metropolitana, Maule, } \\
\text { Biobío y Magallanes; Argentina (Mendoza) y Perú (Lima y Tacna) }\end{array}$ \\
\hline H. Volckmann & 216 & 1850 a 1870 & Desde Atacama (Huasco) hasta Valdivia \\
\hline C. Bertero & 144 & 1828 a 1829 & $\begin{array}{l}\text { Rancagua, Tagua-Tagua, Santiago y Valparaíso (Muñoz-Schick } \\
\text { 1999) }\end{array}$ \\
\hline O. Philippi & 116 & 1887 a 1891 & $\begin{array}{l}\text { Cordillera de las provincias de Cautín, Valdivia y Ranco; Argentina } \\
\text { (Neuquén) }\end{array}$ \\
\hline
\end{tabular}

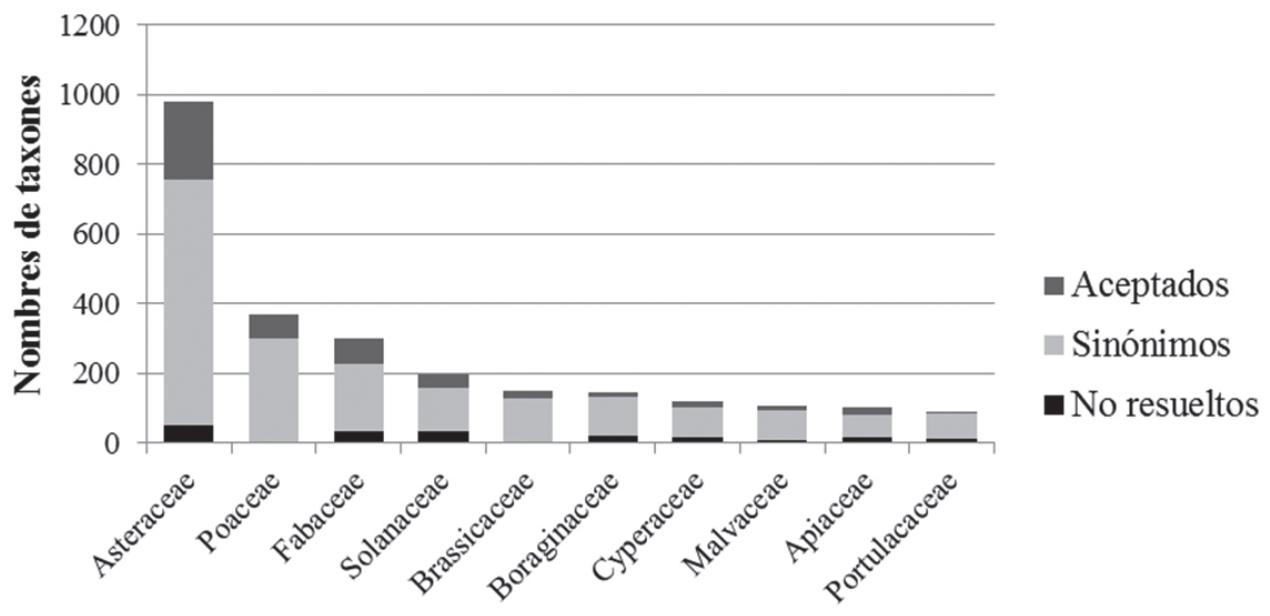

Familias

FIGURA 3. Familias con mayor número de taxones tipo (especies y categorías infraespecíficas) en Herbario SGO.

Figure 3. Families with the highest number of typus taxa (species and infraspecific names) in SGO Herbarium. 
Gayana Bot. 69(1), 2012

TABLA III. Descriptores con mayor número de taxones (especies y categorías infraespecíficas) en el Herbario de tipos SGO.

TABLE III. Descriptors with the highest number of taxa (species and infraspecific names) in the SGO types Herbarium.

\begin{tabular}{lc}
\hline ABREVIACIÓN DESCRIPTORES & NÚMERO DE TAXONES \\
\hline Phil. & 3.360 \\
Reiche & 72 \\
Phil. ex Reiche & 56 \\
Steud. & 54 \\
Clos & 53 \\
J. Remy & 45 \\
F. Phil. & 38 \\
Gay & 31 \\
F.H. Hellwig & 26 \\
E. Desv. & 21 \\
DC. & 19 \\
Skottsb. & 19 \\
Espinosa & 15 \\
Kraenzl. & 15 \\
Griseb. & 12 \\
M. Muñoz & 12 \\
Ravenna & 12 \\
Colla & 11 \\
Leyb. & 11 \\
Bertero ex Colla & 10 \\
\hline
\end{tabular}

\section{CONCLUSIONES}

En sus 182 años de vida, la colección de tipos del herbario SGO se ha incrementado en forma sostenida, a partir de las recolecciones iniciales de C. Bertero en 1828. El período de máxima concentración de tipos es el de 1850-1890, en estrecha relación con la labor de los Philippi, padre e hijo y una serie de colaboradores (incluyendo a Otto Philippi, hijo de Federico). Se incluye ejemplos de etiquetas de los colectores cuyas colecciones fueron reunidas por R.A. Philippi (Fig. 4). Consecuentemente, las localidades con mayor presencia de ejemplares tipo se concentran en las regiones de A tacama y Coquimbo; de ellas, el descubrimiento botánico de la región de Atacama sería responsabilidad de los Philippi, mientras que la flora de Coquimbo fue descrita principalmente por C. Gay. La labor de recolección de los Philippi abarcó incluso territorios limítrofes, especialmente en Argentina; de ahí el importante número de tipos del país vecino. Tanto C. Gay como los Philippi figuran asimismo como los recolectores más importantes de la colección, y les siguen los colaboradores directos del trabajo de los Philippi. En cuanto a la posterior labor de descripción de los taxones, sobresale con mucho R.A. Philippi, seguido muy de lejos por el resto, entre quienes destaca otro notable botánico alemán radicado en Chile como lo fue Carlos Reiche.

El aporte de los Philippi al conocimiento de la biodiversidad de Chile ha sido resaltado en varias ocasiones (Muñoz-Schick 1991, Taylor \& Muñoz-Schick 1994, Marticorena \& Rodríguez 1995, Muñoz-Schick 2003, Castro \& Muñoz-Schick 2004, Castro et al. 2006). Los antecedentes aquí expuestos reafirman nuevamente su inmensa labor, la cual no se remite solamente a la descripción de nuevas especies y la construcción de una de las colecciones más abundantes de Chile, sino que también lograron montar una importante red de colaboradores, quienes en sus viajes de investigación aprovechaban para recolectar las muestras que más tarde serían donadas al museo.

Del análisis de la identidad de los tipos, se desprende que las familias más representadas son las Asteraceae, Poaceae y Fabaceae, lo cual resulta lógico si consideramos que son efectivamente las familias más ricas en especies dentro de la flora de Chile (Moreira-Muñoz 2011). Es notable que además, según la cifra total de especies nativas de Chile, el $54 \%$ de ellas se encuentra representado por la colección de tipos del herbario SGO. Esto significa que el Herbario Nacional conserva las muestras originales para más de la mitad de las especies chilenas que se consideran actualmente válidas. 
A

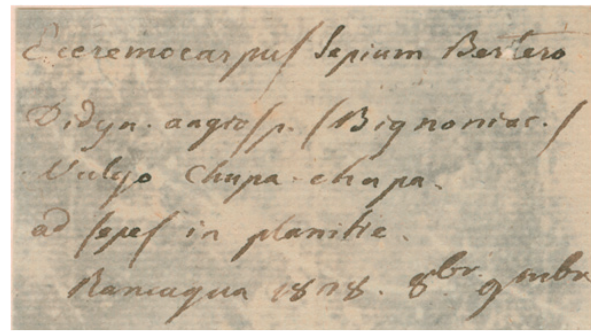

C

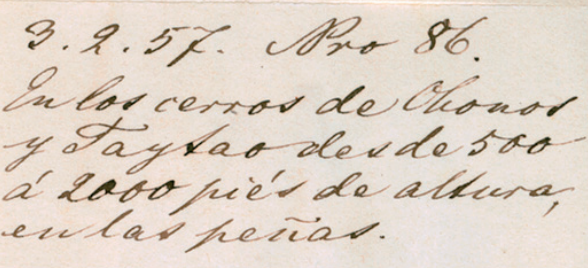

$\mathbf{E}$

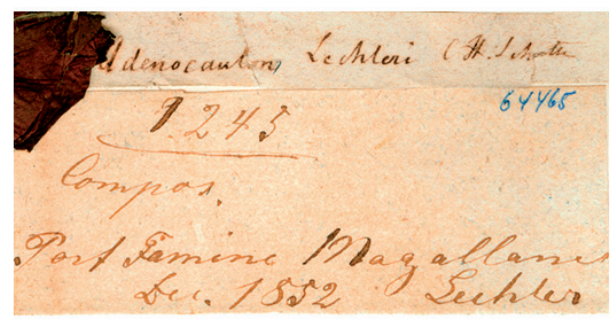

G
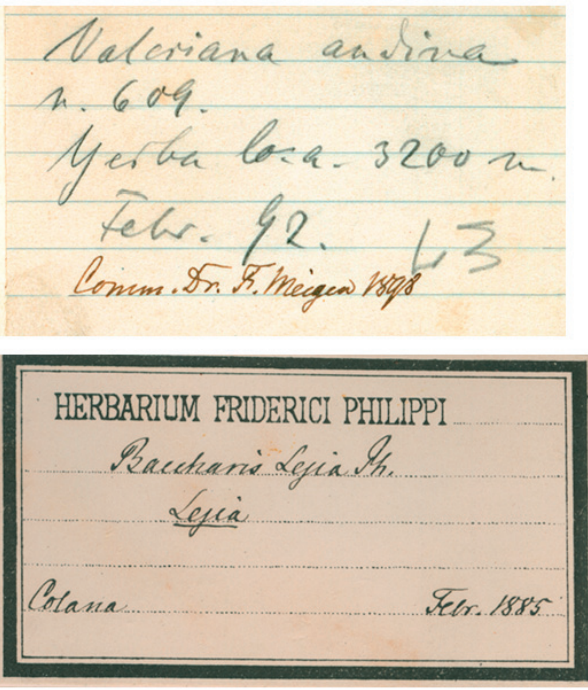

$\mathbf{K}$

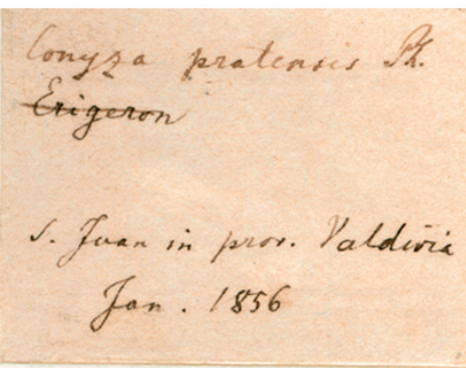

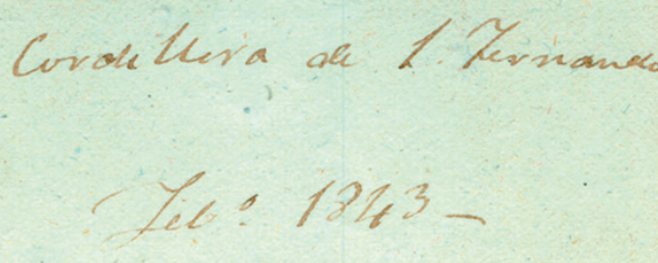

B

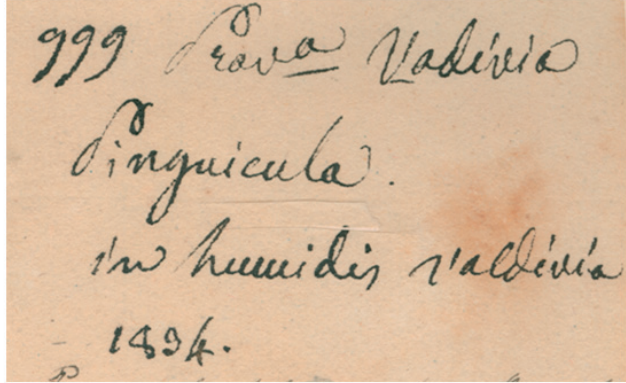

W. Lechler pol. magehlanice. E. R.T. Hohemacter. 1245. Adenocaulon dechleri P.H. Shulta, Bip.n.sp. - Opse. ad portum Dert Famin. Flor. Dec fruct. aprici $m$.

$\mathbf{F}$

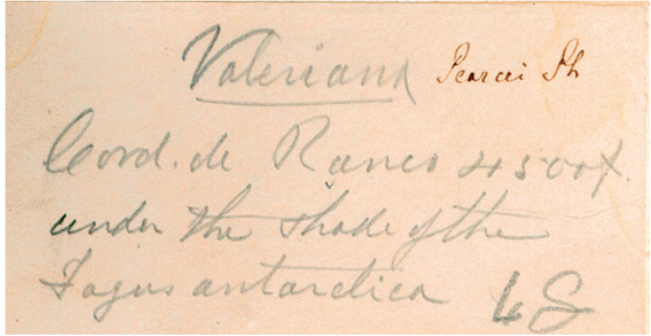

H

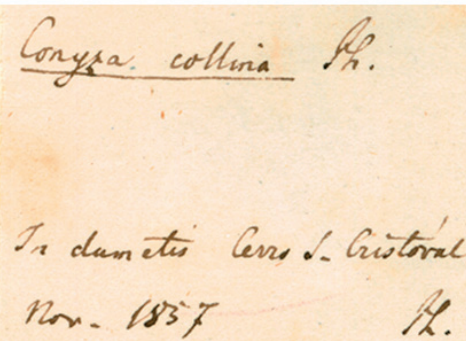

$\mathbf{J}$

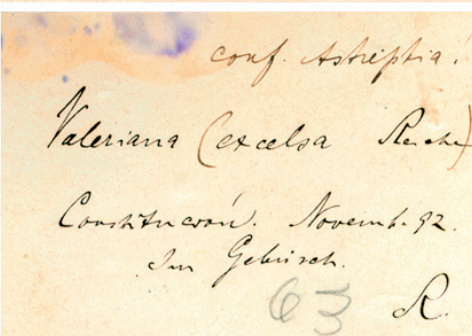

$\mathbf{L}$

A. C. J. Bertero, B. V. Bustillos, C. F. Fonck, D. C. Gay, E. W. Lechler, F. W. Lechler, distribuida por Hohenacker, G. F. Meigen, H. R. Pearce, I. F. Philippi, J. R.A. Philippi, K. R.A. Philippi sin firma, L. C. Reiche.

FIGURA 4. Etiquetas manuscritas de colectores de la colección histórica en SGO.

FIGURE 4. Handwriting of collectors from the historic collection at SGO. 
Es posible mencionar que en la actualidad los herbarios, y específicamente los herbarios nacionales, constituyen colecciones históricas que son un patrimonio nacional y un indicador del desarrollo botánico de un país. Hoy en día su importancia abarca amplios aspectos de la botánica desde la sistemática y la taxonomía hasta la biogeografía, pasando por la conservación de especies, el monitoreo de especies invasoras y los modelos de cambio de rangos de distribución. La colección de tipos SGO, gracias al programa de digitalización, alcanza actualmente estándares de calidad internacionales y se encuentra accesible por vía remota a un amplio público internacional. Dentro de la colección es destacable la presencia de isotipos de autores europeos; la sistematización de la información asociada a los ejemplares tipo sitúa al herbario SGO en una posición estratégica como nodo de interacción con otros herbarios del mundo, lo cual es vital, por ejemplo, para descubrir la presencia de plantas chilenas en herbarios extranjeros. Es necesario enfatizar, en este contexto, el rol que desempeña el personal a cargo de los herbarios, puesto que éstos constituyen colecciones dinámicas que deben estar en proceso continuo de revisión, identificación, montaje y georreferencia. Entre otras cosas, gracias a la adecuada identificación y espacialización de localidades actuales versus históricas, es posible corregir la supuesta presencia de taxones citados erróneamente para Chile.

\section{AGRADECIMIENTOS}

The Andrew W. Mellon Foundation, a través del programa LAPI, ha permitido la digitalización de los ejemplares y la creación de la Base de Datos de la colección de tipos del Herbario SGO. La revisión y georreferencia de la misma contó con el apoyo del proyecto Fondecyt Iniciación $\mathrm{N}^{\mathrm{o}}$ 11085016. A los revisores anónimos por sus comentarios, los que nos estimularon a profundizar algunos aspectos históricos de este trabajo.

\section{BIBLIOGRAFÍA}

Castro, S., A. Camousseight, M. Muñoz-Schick \& F. Jaksic. 2006. Rodulfo Amando Philippi, el naturalista de mayor aporte al conocimiento taxonómico de la diversidad biológica de Chile. Revista Chilena de Historia Natural 79: 133-143.

CAstro, S. \& M. MuÑoz-Schick. 2004. Naturalistas y botánicos: una estimación y caracterización de su aporte al conocimiento de la diversidad florística de Chile. Boletín del Museo Nacional de Historia Natural de Chile 53: 7-16.

Espinosa, O. 1958. La postguerra del Pacífico y la Puna de Atacama (1884-1899). Andrés Bello, Santiago de Chile. 321 pp.

HiRTh, A. 1886. Informe del ayudante de la Comisión exploradora del Río Buta Palena. Anuario Hidrográfico de la Marina de
Chile 11: 176-201

IBAR, E. 1878. Estudios sobre las aguas de Skyring i la parte austral de la Patagonia por el comandante i oficiales de la corbeta Magallanes. Anales de la Universidad de Chile 10: 579637.

Instituto Geográfico Militar (IGM). 1984. Listado de nombres geográficos: desde Visviri (17 $35^{\circ}$ ' latitud sur) a Chaitén ( $42^{\circ} 55^{\prime}$ latitud sur). Instituto Geográfico Militar, Santiago de Chile. Tomo 1, A-M, 1-780 pp. Tomo 2, M-Z, 781$1558 \mathrm{pp}$.

KlingenberG, L. 2007. Monographie der südamerikanischen Gattungen Haplopappus Cass. und Notopappus L. Klingenberg (Asteraceae - Astereae). Bibliotheca Botanica, Heft 157. 331 pp.

Marticorena, C. \& R. Rodríguez (ed.). 1995. Flora de Chile, Vol. 1. Universidad de Concepción. Concepción, Chile. 351 pp.

Moreira-Muñoz, A. 2011. Plant Geography of Chile. Plant and Vegetation, vol.5, Springer, $343 \mathrm{pp}$.

Muñoz Pizarro, C. 1944. El Itinerario de don Claudio Gay. Anales Museo Nacional Historia Natural 22: 27- 44, 8 lám.

Muñoz Pizarro, C. 1960. Las especies de plantas descritas por R.A. Philippi en el siglo XIX. Estudio crítico en la identificación de sus tipos nomenclaturales. Ediciones de la Universidad de Chile, Santiago de Chile. 189 pp.

Muñoz-Pizarro, C. \& E. Pisano. 1950. Botánica. En la Memoria "Siete años de Investigación Agrícola". Departamento de Investigaciones Agrícolas. Santiago de Chile. 309-316.

MuÑoz-Schick, M. 1971. Tipos de plantas descritas por Karl Reiche, conservadas en el Museo Nacional de Historia Natural. Boletín del Museo Nacional de Historia Natural de Chile 32: 377-393.

MuÑoz-Schick, M. 1973. Complemento de "Las Especies de plantas descritas por R.A. Philippi en el siglo XIX". Anales Universidad de Chile, serie 3, 128: 5-69.

MuÑOz-SCHICK, M. 1991. 100 años de la Sección Botánica del Museo Nacional de Historia Natural (1889-1989). Boletín del Museo Nacional de Historia Natural de Chile 42: 181-202.

MuÑoz-Schick, M. 1999. La colección de Carlos José Bertero depositada en el Herbario del Museo Nacional de Historia Natural. Dirección de Bibliotecas Archivos y Museos, Museo Nacional de Historia Natural, Publicación Ocasional $\mathrm{N}^{\mathrm{o}} 53,84 \mathrm{pp}$.

Muñoz-Schick, M. 2003. Importancia de los Philippi en el desarrollo de la botánica chilena. En: M. Larroucau (ed.), El orden prodigioso del mundo natural. Rudolph Amandus Philippi. pp. 41-51. Universidad Austral de Chile y Pehuén Editores, Valdivia, Chile.

MuÑoz-Schick, M. 2007. La colección de plantas vasculares del naturalista Claudio Gay, depositada en el Herbario del Museo Nacional de Historia Natural. Informes FAIPDIBAM 2006. 67-76.

MuÑoz-SCHICK, M. \& O. Prina.1987. Nota aclaratoria sobre especies colectadas por Federico Philippi en su viaje a Tarapacá. Noticiario Mensual, Museo Nacional Historia Natural de Chile 313: 2-17.

PhILIPPI, F. 1865. Excursión botánica en Valdivia desde los Cuncos en el departamento de la Unión, a través de la Cordillera de la Costa, hasta la mar. Anales de la Universidad de Chile 27: 289-302.

PhILIPPI, F. 1885. Reise nach der Provinz Tarapacá. Verhandlungen 
Deutschen Wissenschaftlichen Vereins Santiago 1:135163, 1 mapa.

PhILIPPI, F. 1886. Escursión botánica hecha de orden del Supremo Gobierno en setiembre de 1885 a la provincia de Atacama. Diario Oficial 10 (2793): 1389-1391.

PhiLIPPI, R.A. 1858. Plantarum novarum Chilensium. Linnaea 28: Heft 6: 641-767.

PhilipPI, R.A. 1862. Viaje a los baños i al nuevo Volcán de Chillán. Anales de la Universidad de Chile 20: 279-306.

Philippi, R.A. 1889. Excursión Botánica a la Araucanía efectuada en 1889. Imprenta Lagos, Santiago de Chile. 24 pp.

Riso Patrón, L. 1924. Diccionario Jeográfico de Chile. Imprenta Universitaria, Santiago de Chile. 958 pp.
SAn Román, F. 1892. Carta Jeográfica del Desierto y Cordilleras de Atacama. Dirección de Obras Públicas, Santiago de Chile.

TAYLOR, CH. \& M. Muñoz-SCHICK. 1994. The botanical works of Philippi, father and son, in Chile. Annals of the Missouri Botanical Garden 81: 743-748.

Zuloaga, F. O., O. Morrone \& M.J. Belgrano (eds.). 2008. Catálogo de las Plantas Vasculares del Cono Sur (Argentina, Sur de Brasil, Chile, Paraguay y Uruguay), en Monographs of the Missouri Botanical Garden, No 107 (vol. 1 Pteridophyta, Gymnospermae y Monocotyledoneae; vol. 2 Dicotyledoneae: A-F; vol. 3 Dicotyledoneae: F-Z. $3348 \mathrm{pp}$.

APÉNDICE. Número de taxones totales, válidos, sinónimos y no resueltos.

Appendage. Total number of taxa, valid, synonyms and unresolved.

\begin{tabular}{|c|c|c|c|c|c|}
\hline \multirow{2}{*}{ DIVISIÓN } & \multirow{2}{*}{ FAMILIA* } & \multicolumn{4}{|c|}{ NOMBRES DE TIPOS } \\
\hline & & ACEPTADOS & SINÓNIMOS & No RESUELTOS & TOTAL \\
\hline \multirow[t]{14}{*}{ Pteridophyta } & Aspleniaceae & 0 & 2 & 0 & 2 \\
\hline & Blechnaceae & 2 & 8 & 0 & 10 \\
\hline & Dennstaedtiaceae & 0 & 3 & 0 & 3 \\
\hline & Dryopteridaceae & 4 & 7 & 0 & 11 \\
\hline & Equisetaceae & 0 & 2 & 0 & 2 \\
\hline & Gleicheniaceae & 0 & 2 & 0 & 2 \\
\hline & Hymenophyllaceae & 5 & 5 & 0 & 10 \\
\hline & Isoetaceae & 1 & 0 & 0 & 1 \\
\hline & Lycopodiaceae & 2 & 1 & 0 & 3 \\
\hline & Marsileaceae & 0 & 1 & 0 & 1 \\
\hline & Ophioglossaceae & 2 & 2 & 0 & 4 \\
\hline & Polypodiaceae & 0 & 5 & 0 & 5 \\
\hline & Pteridaceae & 3 & 9 & 0 & 12 \\
\hline & Schizaeaceae & 0 & 1 & 0 & 1 \\
\hline \multirow{22}{*}{$\begin{array}{l}\text { Pinophyta } \\
\text { Magnoliophyta - } \\
\text { Liliopsida }\end{array}$} & Ephedraceae & 4 & 2 & 0 & 6 \\
\hline & Alismataceae & 0 & 1 & 0 & 1 \\
\hline & Alliaceae & 10 & 20 & 6 & 36 \\
\hline & Alstroemeriaceae & 20 & 19 & 6 & 45 \\
\hline & Amaryllidaceae & 9 & 17 & 4 & 30 \\
\hline & Araceae & 0 & 2 & 0 & 2 \\
\hline & Arecaceae & 0 & 1 & 0 & 1 \\
\hline & Asparagaceae & 0 & 2 & 0 & 2 \\
\hline & Bromeliaceae & 6 & 11 & 0 & 17 \\
\hline & Cyperaceae & 19 & 87 & 15 & 121 \\
\hline & Dioscoreaceae & 13 & 12 & 3 & 28 \\
\hline & Hydrocharitaceae & 0 & 1 & 0 & 1 \\
\hline & Iridaceae & 4 & 43 & 0 & 47 \\
\hline & Juncaceae & 1 & 10 & 4 & 15 \\
\hline & Juncaginaceae & 0 & 5 & 2 & 7 \\
\hline & Orchidaceae & 4 & 44 & 14 & 62 \\
\hline & Poaceae & 68 & 295 & 6 & 369 \\
\hline & Potamogetonaceae & 0 & 5 & 0 & 5 \\
\hline & Restionaceae & 0 & 1 & 0 & 1 \\
\hline & Ruppiaceae & 0 & 2 & 0 & 2 \\
\hline & Tecophilaeaceae & 1 & 3 & 0 & 4 \\
\hline & Xanthorrhoeaceae & 1 & 0 & 0 & 1 \\
\hline
\end{tabular}


Gayana Bot. 69(1), 2012

\begin{tabular}{|c|c|c|c|c|c|}
\hline \multirow{2}{*}{ DIVISIÓN } & \multirow{2}{*}{ FAMILIA* } & \multicolumn{4}{|c|}{ NOMBRES DE TIPOS } \\
\hline & & ACEPTADOS & SINÓNIMOS & No RESUELTOS & TOTAL \\
\hline \multirow{51}{*}{$\begin{array}{l}\text { Magnoliophyta - } \\
\text { Magnoliopsida }\end{array}$} & Acanthaceae & 1 & 0 & 0 & 1 \\
\hline & Aizoaceae & 7 & 2 & 1 & 10 \\
\hline & Amaranthaceae & 14 & 35 & 7 & 56 \\
\hline & Anacardiaceae & 0 & 3 & 3 & 6 \\
\hline & Apiaceae & 20 & 67 & 15 & 102 \\
\hline & Apocynaceae & 0 & 14 & 3 & 17 \\
\hline & Araliaceae & 0 & 3 & 0 & 3 \\
\hline & Aristolochiaceae & 0 & 1 & 0 & 1 \\
\hline & Asteraceae & 226 & 703 & 52 & 981 \\
\hline & Atherospermataceae & 0 & 2 & 0 & 2 \\
\hline & Berberidaceae & 2 & 14 & 1 & 17 \\
\hline & Betulaceae & 0 & 1 & 0 & 1 \\
\hline & Bignoniaceae & 4 & 12 & 0 & 16 \\
\hline & Boraginaceae & 15 & 109 & 22 & 146 \\
\hline & Brassicaceae & 19 & 128 & 1 & 148 \\
\hline & Cactaceae & 13 & 20 & 5 & 38 \\
\hline & Calceolariaceae & 23 & 46 & 3 & 72 \\
\hline & Calyceraceae & 8 & 20 & 0 & 28 \\
\hline & Campanulaceae & 0 & 15 & 1 & 16 \\
\hline & Caryophyllaceae & 11 & 44 & 6 & 61 \\
\hline & Celastraceae & 0 & 1 & 0 & 1 \\
\hline & Ceratophyllaceae & 0 & 1 & 0 & 1 \\
\hline & Convolvulaceae & 4 & 13 & 2 & 19 \\
\hline & Crassulaceae & 0 & 7 & 0 & 7 \\
\hline & Cunoniaceae & 0 & 1 & 0 & 1 \\
\hline & Desfontainiaceae & 0 & 1 & 0 & 1 \\
\hline & Elaeocarpaceae & 1 & 2 & 0 & 3 \\
\hline & Elatinaceae & 0 & 1 & 0 & 1 \\
\hline & Ericaceae & 0 & 22 & 4 & 26 \\
\hline & Escalloniaceae & 2 & 24 & 7 & 33 \\
\hline & Euphorbiaceae & 13 & 9 & 0 & 22 \\
\hline & Fabaceae & 73 & 191 & 36 & 300 \\
\hline & Frankeniaceae & 1 & 6 & 0 & 7 \\
\hline & Gentianaceae & 0 & 14 & 3 & 17 \\
\hline & Geraniaceae & 0 & 8 & 0 & 8 \\
\hline & Griseliniaceae & 1 & 2 & 0 & 3 \\
\hline & Grossulariaceae & 3 & 17 & 4 & 24 \\
\hline & Gunneraceae & 3 & 2 & 0 & 5 \\
\hline & Hypericaceae & 0 & 3 & 0 & 3 \\
\hline & Krameriaceae & 0 & 1 & 0 & 1 \\
\hline & Lactoridaceae & 1 & 0 & 0 & 1 \\
\hline & Lamiaceae & 4 & 15 & 2 & 21 \\
\hline & Lauraceae & 0 & 9 & 1 & 10 \\
\hline & Lentibulariaceae & 1 & 0 & 0 & 1 \\
\hline & Linaceae & 0 & 2 & 0 & 2 \\
\hline & Loasaceae & 8 & 19 & 8 & 35 \\
\hline & Loranthaceae & 0 & 1 & 0 & 1 \\
\hline & Lythraceae & 0 & 1 & 4 & 5 \\
\hline & Malesherbiaceae & 7 & 12 & 0 & 19 \\
\hline & Malpighiaceae & 1 & 5 & 0 & 6 \\
\hline & Malvaceae & 12 & 88 & 8 & 108 \\
\hline
\end{tabular}




\begin{tabular}{|c|c|c|c|c|c|}
\hline \multirow{2}{*}{ DivisIÓN } & \multirow{2}{*}{ FAMILIA $*$} & \multicolumn{4}{|c|}{ Nombres de Tipos } \\
\hline & & ACEPTADOS & SINÓNIMOS & No RESUELTOS & TOTAL \\
\hline & Misodendraceae & 2 & 0 & 1 & 3 \\
\hline & Molluginaceae & 0 & 0 & 1 & 1 \\
\hline & Myrtaceae & 1 & 36 & 0 & 37 \\
\hline & Nothofagaceae & 1 & 11 & 1 & 13 \\
\hline & Nyctaginaceae & 0 & 5 & 4 & 9 \\
\hline & Oleaceae & 1 & 0 & 0 & 1 \\
\hline & Onagraceae & 6 & 28 & 2 & 36 \\
\hline & Orobanchaceae & 7 & 4 & 5 & 16 \\
\hline & Oxalidaceae & 14 & 43 & 3 & 60 \\
\hline & Phrymaceae & 2 & 11 & 0 & 13 \\
\hline & Phytolaccaceae & 0 & 1 & 2 & 3 \\
\hline & Piperaceae & 2 & 1 & 0 & 3 \\
\hline & Plantaginaceae & 9 & 34 & 12 & 55 \\
\hline & Plumbaginaceae & 0 & 7 & 0 & 7 \\
\hline & Polemoniaceae & 1 & 12 & 7 & 20 \\
\hline & Polygalaceae & 3 & 4 & 2 & 9 \\
\hline & Polygonaceae & 11 & 16 & 9 & 36 \\
\hline & Portulacaceae & 1 & 74 & 12 & 87 \\
\hline & Primulaceae & 0 & 4 & 2 & 6 \\
\hline & Ranunculaceae & 4 & 24 & 5 & 33 \\
\hline & Rhamnaceae & 0 & 11 & 3 & 14 \\
\hline & Rosaceae & 4 & 25 & 3 & 32 \\
\hline & Rubiaceae & 13 & 21 & 0 & 34 \\
\hline & Salicaceae & 0 & 7 & 0 & 7 \\
\hline & Santalaceae & 7 & 8 & 8 & 23 \\
\hline & Saxifragaceae & 0 & 0 & 1 & 1 \\
\hline & Scrophulariaceae & 0 & 2 & 0 & 2 \\
\hline & Solanaceae & 39 & 126 & 34 & 199 \\
\hline & Tetrachondraceae & 1 & 0 & 0 & 1 \\
\hline & Thymelaeaceae & 0 & 2 & 0 & 2 \\
\hline & Tropaeolaceae & 5 & 7 & 0 & 12 \\
\hline & Urticaceae & 3 & 3 & 0 & 6 \\
\hline & Valerianaceae & 12 & 27 & 3 & 42 \\
\hline & Verbenaceae & 4 & 51 & 3 & 58 \\
\hline & Violaceae & 31 & 16 & 7 & 54 \\
\hline & Vivianiaceae & 2 & 2 & 7 & 11 \\
\hline & Winteraceae & 1 & 1 & 0 & 2 \\
\hline & Zygophyllaceae & 1 & 3 & 0 & 4 \\
\hline & Total & 865 & 2975 & 396 & 4236 \\
\hline & $\%$ & 20,4 & 70,2 & 9,3 & 100,0 \\
\hline
\end{tabular}

*Los tipos han sido clasificados según las familias indicadas en Moreira-Muñoz, 2011. / The type names has been classified according to the families indicated in Moreira-Muñoz, 2011.

Recibido: 19.05.11

Aceptado: 18.10.11 